\title{
Pierre Bourdieu: Una mirada etnográfica Aportes al diseño sobre la cultura material y la educación Proyecto de Investigación $\mathrm{N}^{\circ} 15.1$
}

Equipo de Investigación ${ }^{(1)}$

Patricia Vargas y Laura Colabella

por Facultad de Diseño y Comunicación, Universidad de Palermo (ARG) y el Centro de Antropología Social del Instituto de Desarrollo Económico y Social, CAS-IDES (ARG)

\begin{abstract}
Resumen: El Proyecto de Investigación 15.1 es un homenaje al sociólogo francés Pierre Bourdieu, uno de los autores más citados en las ciencias sociales, a través de artículos de investigadores argentinos que retoman libros o conceptos específicos del autor, tributo para repensar sus propios temas de investigación. Específicamente se recuperan los aportes bourdeanos desde una óptica conceptual y metodológica, con especial énfasis en el trabajo de campo y el proceso de reflexividad, en investigaciones empíricas y/o etnográficas, que dan cuenta de la contribución de Pierre Bourdieu en sus procesos de producción de conocimiento social, en particular en los campos del Diseño, la educación y las clases sociales.
\end{abstract}

Palabras claves: Bourdieu - Diseño - Clases Sociales - Educación

[Resúmenes en inglés y portugués en la página 196]

${ }^{(1)}$ Los CVs del Equipo de Investigación pueden consultarse en el Capítulo Directores de Líneas y Coordinadores de Proyectos de esta misma Edición.

\section{Acerca del Proyecto 15.1}

Pierre Bourdieu: Una mirada etnográfica

Aportes al diseño sobre la cultura material y la educación

El Proyecto 15.1 Pierre Bourdieu: Una mirada etnográfica. Aportes al diseño sobre la cultura material y la educación, indaga sobre los aportes al Diseño desde las perspectivas 
de Pierre Bourdieu y visibiliza los trabajos de investigación que articulan las investigaciones del sociólogo con material empírico acerca de la cultura material, el diseño y la educación.

Se acordó entre la Universidad de Palermo y el Centro de Antropología Social del Instituto de Desarrollo Económico y Social (CAS - IDES, Argentina), avanzar en una investigación en la que participan académicos de ambas Instituciones, con la coordinación compartida de Patricia Vargas y Laura Colabella (UP y CAS-IDES), con el fin de generar un debate en cómo la teoría y la práctica sociológica, según el pensamiento de Pierre Bourdieu, entrecruza tramas, lenguajes y medios, para arribar a reflexiones en las que se conjugue su vínculo con la etnografía, la cultura material y la educación. Las reflexiones y los resultados obtenidos en el Proyecto 15.1 Pierre Bourdieu: Una mirada etnográfica. Aportes al diseño sobre la cultura material y la educación, se continúan desarrollando en los Proyectos actualmente en curso de la misma Línea de Investigación y bajo las mismas Directoras Patricia Vargas y Laura Colabella.

El principal objetivo es:

- Visibilizar los trabajos de investigación que articulan los aportes del sociólogo Pierre Bourdieu y Annete Weiner con material empírico acerca de la cultura material, el diseño y la educación.

\section{Acerca de la Línea 15}

Etnografía, Cultura material y Educación. Reflexiones y aportes al Diseño dirigida por Patricia Vargas y Laura Colabella se desarrolla de manera ininterrumpida desde 2016 en la Facultad de Diseño y Comunicación (UP, Argentina), e incluye hasta el momento un proyecto finalizado el 15.1 Pierre Bourdieu: Una mirada etnográfica. Aportes al diseño sobre la cultura material y la educación, coordinado por Patricia Vargas y Laura Colabella (UP, CAS-IDES).

\section{Mapa de Áreas y Proyectos}

El Proyecto 15.1 se vincula con todas las carreras de grado y posgrado correspondientes a la Facultad de Diseño y Comunicación, dado que impacta en la incorporación de la Investigación en la lógica de la docencia universitaria.

\section{Productos y Resultados}

\section{a)- Publicaciones}

Cuaderno del Centro de Estudios de Diseño y Comunicación No88. (2021) Aportes al análisis de las prácticas culturales contemporáneas de la Argentina reciente, desde la pers- 
pectiva de Pierre Bourdieu. Coordinación Laura Colabella y Patricia Vargas (UP - CAS IDES). Facultad de Diseño y Comunicación Año XXI, Febrero 2021, Buenos Aires, Argentina. ISSN: 1668-0227. Esta publicación documenta y comunica los resultados alcanzados en el proyecto 15.1 Pierre Bourdieu: una mirada etnográfica. A continuación se detallan los autores y artículos contenidos en ella:

Vargas Patricia, Colabella Laura (2021) Prólogo. Aportes al análisis de las prácticas culturales contemporáneas de la Argentina reciente, desde la perspectiva de Pierre Bourdieu (Pp. 11-16)

Colabella Laura, Vargas Patricia (2021) Bourdieu en el conurbano: un viaje de ida y vuelta. Alteridad y trabajo de campo antropológico en la UNAJ y la UNPAZ (Pp. 51-71)

Dukuen Juan (2021) Un arte de inventar: el habitus en la lectura bourdiana de Panofsky (Pp. 17-34)

Gessaghi Victoria, Méndez Alicia (2021) La Nobleza de Estado, algunas reflexiones a partir del trabajo de campo con elites educativas en la Argentina (Pp. 35-49) Schiava D'Albano Lorena N (2021) "Solo hay un camino entre la persona que eres y la que quieres ser". Análisis de un curso de imagen bajo la óptica de El baile de los solteros (Pp. 73-86)

Gutiérrez Alicia B, Mansilla Héctor O (2021) La dialéctica entre lo objetivo y lo vivido: el análisis de la desigualdad social en Córdoba, Argentina (Pp. 87-109)

Blanco Esmoris María Florencia (2021) ¿La indeterminación del orden binario? Notas descriptivas para pensar "La casa o el mundo dado vuelta" de Pierre Bourdieu en una etnografía contemporánea (Pp. 111-127)

Miguel Paula (2021) El "diseño" como valor y la conformación de un universo de creencias (Pp. 129-142)

Correa María Eugenia (2021) La lucha por la legitimidad. Estudio del campo profesional del Diseño Industrial a la luz de la teoría de Pierre Bourdieu (Pp. 143-158) Guerschman Bárbara (2021) Aprender a verse como una marca. El cálculo, tiempo y ethos en Argelia 60 (Pp. 177-192)

Correa María Eugenia, Romani Matías J (2021) El lujo tecnológico. Distinción y esnobismo en un contexto de prohibición: el consumo de Apple en Argentina (2015-2018) (Pp. 177-192)

Alatsis Gabriela C (2021) El rol de los intermediarios culturales en la producción de la "creencia colectiva": la conformación de un circuito de diseño en Quilmes (Pp. 193 a 205)

Viotti Nicolás, Irisarri Victoria (2021) ¿ Más allá de la distinción? La reproducción de diferencias sociales y los regímenes de objetos-personas (Página 207-223)

\section{b)- Congresos / Coloquios / Plenarios}

$3^{\circ}$ Plenario de Directores de Investigación DC. 26, 27 y 29 de agosto de 2019. En este tercer plenario los Directores de las Líneas y Proyectos de Investigación presentaron a sus pares y al conjunto del Programa de Investigación de la Facultad de Diseño y Comuni- 
cación, los resultados obtenidos y/o en proceso (publicaciones y acuerdos), junto con los avances de Proyectos. La Directora de la Línea de Investigación №15 presentó a sus pares, los resultados del proyecto 15.1 Pierre Bourdieu: una mirada etnográfica y anticipó las gestiones y avances del segundo proyecto de la Línea.

IV Coloquio de Investigación y Desarrollo en Diseño Latino. Universidad de Palermo, 29 de julio de 2019. X Congreso Latinoamericano de Enseñanza del Diseño. Semana Internacional del Diseño en Palermo.

En la comisión Etnografía, Cultura material y Educación se presentaron las conclusiones del proyecto 15.1 Pierre Bourdieu: una mirada etnográfica.

Se expusieron parte de los artículos que componen el Cuaderno 88: Aportes al análisis de las prácticas culturales contemporáneas de la Argentina reciente, desde la perspectiva de Pierre Bourdieu. Expusieron: Laura Colabella, Patricia Vargas, Lorena N. Schiava D’Albano, Victoria Gessaghi, Alicia Méndez, Bárbara Guerschman, María Eugenia Correa, Gabriela C. Alatsis, María Eugenia Correa y Matías J. Romani.

A continuación se detallan las ponencias presentadas en esta Comisión coordinada por las Directoras del proyecto Laura Colabella y Patricia Vargas

Laura Colabella y Patricia Vargas (Argentina) - Coordinadoras de la Comisión

Bourdieu en el conurbano: un viaje de ida y vuelta. Alteridad y trabajo de campo antropológico en la UNAJ y la UNPAZ

Lorena N. Schiava D'Albano (Argentina)

"Solo hay un camino entre la persona que eres y la que quieres ser". Análisis de un curso de Imagen bajo la óptica de El baile de los solteros

Victoria Gessaghi y Alicia Méndez (Argentina)

La Nobleza de Estado, algunas reflexiones a partir del trabajo de campo con elites educativas en la Argentina

Bárbara Guerschman (Argentina)

Aprender a verse como una marca. El cálculo, tiempo y ethos en Argelia 60

María Eugenia Correa (Argentina)

La lucha por la legitimidad. Estudio del campo profesional del diseño Industrial a la luz de la teoría de Pierre Bourdieu

Gabriela C. Alatsis (Argentina)

El rol de los intermediarios culturales en la producción de la "creencia colectiva": la conformación de un circuito de diseño en Quilmes

María Eugenia Correa y Matías J. Romani (Argentina)

El lujo tecnológico. Distinción y esnobismo en un contexto de prohibición: el consumo de Apple en Argentina (2015-2018)

$2^{\circ}$ Plenario de Directores de Investigación DC, 27 de agosto y 4 de septiembre - 2018. Es este segundo plenario los Directores de las Líneas y Proyectos de Investigación presentaron a sus pares y al conjunto del Programa de Investigación de la Facultad de Diseño y 
Comunicación, los resultados obtenidos y/o en proceso (publicaciones y acuerdos), junto con los avances de Proyectos. Las Directoras de la Línea de Investigación No 15 expusieron las reflexiones del comienzo del Proyecto 15.1 Pierre Bourdieu: una mirada etnográfica (finalizado).

III Coloquio de Investigación y Desarrollo en Diseño Latino. Universidad de Palermo, 30 de julio de 2018. IX Congreso Latinoamericano de Enseñanza del Diseño. Semana Internacional del Diseño en Palermo.

En la comisión Etnografía, Cultura material y Educación se presentaron las reflexiones y avances del proyecto 15.1 Pierre Bourdieu: una mirada etnográfica. Expusieron: Gabriela Alatsis, Maria Florencia Blanco Esmoris, Eugenia Correa, Juan Dukuen, Victoria Gessaghi, Alicia Mendez, Barbara Guersham, Alicia Gutierrez, Paula Miguel, Lorena Schiava DÁlbano, Juan pablo Vazquez Gutierrez y Nicolas Viotti.

A continuación se detallan las ponencias presentadas en esta Comisión coordinada por las Directoras del proyecto Patricia Vargas y Laura Colabella:

Gabriela Alatsis

El rol de los intermediarios culturales en la producción de la "creencia colectiva": la conformación de un circuito de diseño en Quilmes

María Florencia Blanco Esmoris

¿La indeterminación del orden binario?: apuntes para pensar "La casa o el mundo dado vuelta" de Pierre Bourdieu en una etnografía contemporánea

Eugenia Correa

La lucha por la legitimidad. Estudio del campo profesional del Diseño Industrial a la luz de la teoría de Pierre Bourdieu

Juan Dukuen

Un arte de inventar: el habitus en la lectura bourdiana de Panofsky

Victoria Gessaghi y Alicia Mendez

La nobleza de estado de Pierre Bourdieu en dos investigaciones sobre educación

Bárbara Guerschman

El cálculo y el tiempo en Argelia 60. Aprender a verse como una marca

\section{Alicia Gutiérrez}

La dialéctica entre lo objetivo y lo vivido: las dimensiones subjetivas de la dominación

Paula Miguel

El diseño como valor y la conformación de un universo de creencia

Lorena Schiava D 'Albano

"Sólo hay un camino entre la persona que eres y la que quieres ser". Análisis de un curso de imagen bajo la óptica de El baile de los solteros

Juan Pablo Vazquez Gutiérrez

Diversidad cultural e inclusión en el espacio escolar. Retos y preguntas en torno a lo intercultural a la luz de una experiencia en el nivel superior 


\title{
Nicolás Viotti
}

El problema de la distinción socio-cultural. Bourdieu y la materialidad

\section{c)- Formación de Posgrado e Impacto curricular}

Las Directoras Patricia Vargas y Laura Colabella son Docentes de Posgrado en Diseño de la Facultad de Diseño y Comunicación: Maestría en Gestión del Diseño y Doctorado en Diseño, e incorporan los contenidos de su investigación a sus asignaturas de posgrado.

\begin{abstract}
The Research Project 15.1 is a tribute to the French sociologist Pierre Bourdieu, one of the most cited authors in the social sciences, through articles by Argentine researchers that take up books or specific concepts of the author, a tribute to rethink their own themes of investigation. Specifically, the Bourdieu contributions are recovered from a conceptual and methodological perspective, with special emphasis on field work and the process of reflexivity, in empirical and / or ethnographic investigations, which account for the contribution of Pierre Bourdieu in his production processes of social knowledge, particularly in the fields of Design, education and social classes.
\end{abstract}

Keywords: Pierre Bourdieu - Design - Social Classes - Education

Resumo: O Projeto de Pesquisa 15.1 é uma homenagem ao sociólogo francês Pierre Bourdieu, um dos autores mais citados nas ciências sociais, por meio de artigos de pesquisadores argentinos que abordam livros ou conceitos específicos do autor, uma homenagem para repensar seus próprios temas de investigação. Especificamente, as contribuições de Bourdieu são recuperadas de uma perspectiva conceitual e metodológica, com especial ênfase no trabalho de campo e no processo de reflexividade, em investigações empíricas e / ou etnográficas, que dão conta da contribuição de Pierre Bourdieu em seus processos de produção de conhecimento social, nomeadamente nas áreas do Design, educação e classes sociais.

Palavras chave: Pierre Bourdieu - Design - Classes sociais - Educação

[Las traducciones de los resúmenes fueron realizadas a través de traductor automático] 